VoL. $70(2004)$ [293-299]

\title{
NORM OF COMPOSITION OPERATORS ON THE BLOCH SPACE
}

\section{Chengu Xiong}

\begin{abstract}
The author estimates the semi-norm and norm of composition operators on the Block space; and obtains several necessary conditions for a composition operator to be isometric.
\end{abstract}

\section{INTRODUCTION}

Let $\mathbf{D}$ be the unit disk in the complex plane $\mathbb{C}$ and $H(\mathbf{D})$ be the set of all analytic functions on $\mathbf{D}$. A function $f \in H(\mathrm{D})$ is said to be a Bloch function if it satisfies

$$
\|f\|_{\beta}=\sup _{z \in \mathrm{D}}\left(1-|z|^{2}\right)\left|f^{\prime}(z)\right|<\infty .
$$

The set of all Bloch functions is called the Bloch space, which becomes a Banach space [1] under the norm

$$
\|f\|_{B}=|f(0)|+\|f\|_{\beta}=|f(0)|+\sup _{z \in \mathbf{D}}\left(1-|z|^{2}\right)\left|f^{\prime}(z)\right| .
$$

Let $\varphi$ be a holomorphic mapping of $\mathrm{D}$ into itself. It is known that $\varphi$ induces a bounded composition operator $C_{\varphi}(f)=f \circ \varphi$ on the Bloch space ([4]). The semi-norm and norm of $C_{\varphi}$ are defined as

$$
\left\|C_{\varphi}\right\|_{\beta}=\sup _{\|f\|_{\mathcal{B}}=1}\|f \circ \varphi\|_{\beta} \quad \text { and } \quad\left\|C_{\varphi}\right\|_{B}=\sup _{\|f\|_{B}=1}\|f \circ \varphi\|_{\mathcal{B}}
$$

respectively.

If $\left\|C_{\varphi}(f)\right\|_{\mathcal{B}}=\|f\|_{\mathcal{B}}$ for every $f \in \mathcal{B}$, then $C_{\varphi}$ is called an isometric operator on the Bloch space.

On the Hardy space $H^{2}$, Littlewood [2] showed that

$$
\left\|C_{\varphi}\right\|_{H^{2}} \leqslant \sqrt{\frac{1+|\varphi(0)|}{1-|\varphi(0)|}} .
$$

Received 31st March, 2004

This research is supported in part by the National Science Foundation of China (NO. 10371069).

Copyright Clearance Centre, Inc. Serial-fee code: 0004-9727/04 \$A2.00+0.00. 
If $\varphi$ is an inner function, Nordgren [3] showed that

$$
\left\|C_{\varphi}\right\|_{H^{2}}=\sqrt{\frac{1+|\varphi(0)|}{1-|\varphi(0)|}}
$$

Moreover, $C_{\varphi}$ is isometric if and only if $\varphi$ is an inner function with $\varphi(0)=0$. If $\varphi(0) \neq 0$, Shapiro [5] showed that

$$
\left\|C_{\varphi}\right\|_{H^{2}}=\sqrt{\frac{1+|\varphi(0)|}{1-|\varphi(0)|}}
$$

if and only if $\varphi$ is an inner function.

In this paper, we consider similar problems on the Bloch space. In Section 2, we estimate the semi-norm and norm of composition operators. In Section 3, we obtain several necessary conditions for a composition operator to be isometric. Finally, we propose a question.

\section{SEMI-NORM AND NORM OF COMPOSITION OPERATORS}

Denote

$$
\tau_{\varphi}(z)=\frac{1-|z|^{2}}{1-|\varphi(z)|^{2}}\left|\varphi^{\prime}(z)\right|, \quad \tau_{\varphi}^{\infty}=\sup _{z \in \mathbf{D}} \tau_{\varphi}(z) .
$$

By Schwarz-Pick Lemma, $\tau_{\varphi}(z) \leqslant 1$ and $\tau_{\varphi}^{\infty} \leqslant 1$.

THEOREM 1. Let $\varphi$ be a holomorphic mapping of $\mathbf{D}$ into itself, then the seminorm

$$
\left\|C_{\varphi}\right\|_{\beta}=\sup _{\|f\|_{B}=1}\left(1-|z|^{2}\right)\left|f^{\prime}(\varphi(z)) \varphi^{\prime}(z)\right|=\tau_{\varphi}^{\infty} .
$$

Proof: By the definition,

$$
\begin{aligned}
\left\|C_{\varphi}\right\|_{\beta} & =\sup _{\|f\|_{B}=1}\left(1-|z|^{2}\right)\left|f^{\prime}(\varphi(z)) \varphi^{\prime}(z)\right| \\
& =\sup _{\|f\|_{B}=1}\left(1-|\varphi(z)|^{2}\right)\left|f^{\prime}(\varphi(z))\right| \cdot \frac{1-|z|^{2}}{1-|\varphi(z)|^{2}}\left|\varphi^{\prime}(z)\right| \leqslant \tau_{\varphi}^{\infty}
\end{aligned}
$$

On the other hand, let

$$
F(z)=\frac{1}{2} \log \frac{1+z}{1-z}, \quad F(0)=0, \quad z \in \mathrm{D},
$$

then $F \in \mathcal{B},\|F\|_{\mathcal{B}}=1$ and $\left(1-|z|^{2}\right)\left|F^{\prime}(z)\right|=1$ for $z \geqslant 0$. For any $\varepsilon>0$, there exists a $z_{0} \in \mathbf{D}$ such that $\tau_{\varphi}\left(z_{0}\right)>\tau_{\varphi}^{\infty}-\varepsilon$. Let $\varphi\left(z_{0}\right)=e^{i \theta}\left|\varphi\left(z_{0}\right)\right|, g(z)=F\left(e^{-i \theta} z\right)$, then

$$
\begin{aligned}
\left\|C_{\varphi}\right\|_{\beta} \geqslant\left\|C_{\varphi}(g)\right\|_{\beta} & \geqslant \sup _{z \in \mathbf{D}}\left(1-|\varphi(z)|^{2}\right)\left|F^{\prime}\left(e^{-i \theta} \varphi(z)\right)\right| \cdot \frac{1-|z|^{2}}{1-|\varphi(z)|^{2}}\left|\varphi^{\prime}(z)\right| \\
& \geqslant\left(1-\left|\varphi\left(z_{0}\right)\right|^{2}\right)\left|F^{\prime}\left(e^{-i \theta} \varphi\left(z_{0}\right)\right)\right| \cdot \frac{1-\left|z_{0}\right|^{2}}{1-\left|\varphi\left(z_{0}\right)\right|^{2}}\left|\varphi^{\prime}\left(z_{0}\right)\right| \\
& =\tau_{\varphi}\left(z_{0}\right)>\tau_{\varphi}^{\infty}-\varepsilon
\end{aligned}
$$


For the arbitrariness of $\varepsilon$, we have $\left\|C_{\varphi}\right\|_{\beta} \geqslant \tau_{\varphi}^{\infty}$. The proof is completed.

COROLlaRY 1. Let $\varphi$ be a holomorphic mapping of $\mathrm{D}$ into itself, then

$$
\left\|C_{\varphi}\right\|_{\mathcal{B}} \geqslant \max \left\{1, \frac{1}{2} \log \frac{1+|\varphi(0)|}{1-|\varphi(0)|}\right\}
$$

PROoF: As in the proof of theorem 1, let $\varphi(0)=e^{i \theta}|\varphi(0)|$,

$$
F(z)=\frac{1}{2} \log \frac{1+z}{1-z} \quad F(0)=0,
$$

and $g(z)=F\left(e^{-i \theta} z\right)$. We have

$$
\left\|C_{\varphi}\right\|_{B} \geqslant\|g(\varphi)\|_{B} \geqslant|g((\varphi(0)))| \geqslant \frac{1}{2} \log \frac{1+|\varphi(0)|}{1-|\varphi(0)|} .
$$

Taking $f(z)=1$, then $\left\|C_{\varphi}(f)\right\|_{\mathcal{B}}=1$ and $\left\|C_{\varphi}\right\|_{\mathcal{B}} \geqslant 1$. The proof is completed.

LEMma 1 . If $f \in \mathcal{B}$, then

$$
|f(z)| \leqslant|f(0)|+\frac{\|f\|_{\mathcal{B}}-|f(0)|}{2} \log \frac{1+|z|}{1-|z|} .
$$

Proof: By the definition,

$$
\|f\|_{\mathcal{B}}=|f(0)|+\sup _{z \in \mathbf{D}}\left(1-|z|^{2}\right)\left|f^{\prime}(z)\right| .
$$

Hence

$$
\begin{aligned}
\left|f^{\prime}(z)\right| & \leqslant \frac{\|f\|_{\mathcal{B}}-|f(0)|}{1-|z|^{2}}, \\
|f(z)| & =\left|f(0)+\int_{0}^{z} f^{\prime}(z) d z\right| \leqslant|f(0)|+\int_{0}^{|z|}\left|f^{\prime}(z)\right| d|z| \\
& \leqslant|f(0)|+\frac{\|f\|_{B}-|f(0)|}{2} \log \frac{1+|z|}{1-|z|} .
\end{aligned}
$$

THEOREM 2. Let $\varphi$ be a holomorphic mapping of $\mathrm{D}$ into itself, then

$$
\left\|C_{\varphi}\right\|_{\mathcal{B}} \leqslant \max \left\{1, \frac{1}{2} \log \frac{1+|\varphi(0)|}{1-|\varphi(0)|}+\tau_{\varphi}^{\infty}\right\} .
$$

In particular, if $\varphi(0)=0$, then $\left\|C_{\varphi}\right\|_{\mathcal{B}}=1$.

Proof: Taking $f \in \mathcal{B}$ with $\|f\|_{\mathcal{B}}=1$, we have

$$
\begin{aligned}
\left\|C_{\varphi}(f)\right\|_{B} & =|f(\varphi(0))|+\sup _{z \in \mathbf{D}}\left(1-|z|^{2}\right)\left|f^{\prime}(\varphi(z))\right|\left|\varphi^{\prime}(z)\right| \\
& =|f(\varphi(0))|+\sup _{z \in \mathbf{D}}\left(1-|\varphi(z)|^{2}\right)\left|f^{\prime}(\varphi(z))\right| \cdot \frac{1-|z|^{2}}{1-|\varphi(z)|^{2}}\left|\varphi^{\prime}(z)\right| \\
& \leqslant|f(\varphi(0))|+\left(\|f\|_{B}-|f(0)|\right) \tau_{\varphi}^{\infty} \\
& =|f(\varphi(0))|+(1-|f(0)|) \tau_{\varphi}^{\infty} .
\end{aligned}
$$


Using lemma 1 ,

$$
|f(\varphi(0))| \leqslant|f(0)|+\frac{1-|f(0)|}{2} \log \frac{1+|\varphi(0)|}{1-|\varphi(0)|} .
$$

Notice that $0 \leqslant|f(0)| \leqslant 1$, then

$$
\begin{aligned}
\left\|C_{\varphi}(f)\right\|_{\mathcal{B}} & \leqslant|f(0)|+(1-|f(0)|)\left(\frac{1}{2} \log \frac{1+|\varphi(0)|}{1-|\varphi(0)|}+\tau_{\varphi}^{\infty}\right) \\
& \leqslant \max \left\{1, \frac{1}{2} \log \frac{1+|\varphi(0)|}{1-|\varphi(0)|}+\tau_{\varphi}^{\infty}\right\} .
\end{aligned}
$$

The proof is completed.

To deal with $\tau_{\varphi}^{\infty}$, we need several concepts which can be found in [6]. Let $w \in T$ $=\partial \mathrm{D}$ and $0<\alpha<\pi / 2$. The Stolz angle at $w$, denote by $S(w, \alpha)$, is the region between two straight lines in $\mathbf{D}$ that meet at $w$ with angle $2 \alpha$ and are symmetric about the radius to $w$. For $f \in H(\mathbf{D}), w \in T$ and a constant $L \in \mathbb{C}$, then

$$
\angle \lim _{z \rightarrow w}=L
$$

means that $f(z) \rightarrow L$ as $z \rightarrow w$ through any Stolz angle at $w$. In this case, we say $L$ is the angular limit of $f$ at $w$. Suppose $\varphi$ is a holomorphic mapping of $\mathbf{D}$ into itself and $w \in T$. If there exists a constant $\eta \in T$ such that

$$
\angle \lim _{z \rightarrow w} \frac{\varphi(z)-\eta}{z-w}
$$

exists finitely, then we say this limit is the angle derivative of $\varphi$ at $w$ and denote it by $\varphi^{\prime}(w)$

We need the following.

THE JUlia-Carathéodory Theorem. ([6].) Suppose $\varphi$ is a holomorphic self-map of $\mathbf{D}$, and $w \in T$. Then the following three statements are equivalent:

(1) $\liminf _{z \rightarrow w} \frac{1-|\varphi(z)|}{1-|z|}=\delta<\infty$;

(2) $\angle \lim _{z \rightarrow w} \frac{\eta-\varphi(z)}{w-z}$ exists for some $(\eta \in T)$;

(3) $\angle \lim _{z \rightarrow w} \varphi^{\prime}(z)$ exists, and $\lim _{z \rightarrow w} \angle \varphi(z)=\eta$.

Moreover, if one of the above conditions holds, then $\delta>0$, the $\eta$ in (1) and (2) are the same, and the limit in (2) coincides with that of the derivative in (3), with both equal $\bar{w} \eta \delta$.

LEMMA 2. Suppose $\varphi$ is a holomorphic self-map of $\mathrm{D}$ and has an angle derivative at some point of $T=\partial \mathbf{D}$. Then

$$
\tau_{\varphi}^{\infty}=1
$$


Proof: By Theorems 1,2 and the Schwarz-Pick lemma, we need only prove $\tau_{\varphi}^{\infty} \geqslant 1$. Assume that $\varphi$ has an angle derivative $\mu$ at the point $w \in T$. Using the Julia-Carathéodory theorem for $\varphi$, then there exist $\delta>0$ and $\eta \in T$ such that $\mu=\angle \lim _{z \rightarrow w} \varphi^{\prime}(z)=\bar{w} \eta \delta$ and

$$
\liminf _{z \rightarrow w} \frac{1-|\varphi(z)|}{1-|z|}=\delta
$$

Hence

$$
\angle \lim _{z \rightarrow w} \tau_{\varphi}=\angle \lim _{z \rightarrow w} \frac{1-|z|^{2}}{1-|\varphi(z)|^{2}}\left|\phi^{\prime}(z)\right|=1
$$

and $\left\|\tau_{\varphi}\right\|_{\infty} \geqslant 1$. The proof is completed.

By Theorem 1, 2, Corollary of the Theorem 1 and the Lemma 2, we have following.

Corollary 2. Suppose that $\varphi$ is a holomorphic self-map of $D$ and has an angle derivative at some point of $T=\partial \mathrm{D}$. Then

$$
\begin{gathered}
\left\|C_{\varphi}\right\|_{\beta}=1, \\
\max \left\{1, \frac{1}{2} \log \frac{1+|\varphi(0)|}{1-|\varphi(0)|}\right\} \leqslant\left\|C_{\varphi}\right\|_{B} \leqslant 1+\frac{1}{2} \log \frac{1+|\varphi(0)|}{1-|\varphi(0)|} .
\end{gathered}
$$

Moveover, for any $a \in[0,1)$, the left equality of the above ineqality can hols for $\varphi(z)=a$ and the right for $\varphi(z)=(a-z) /(1-\bar{a} z)$.

\section{ISOMETRIC COMPOSITION OPERATORS}

LEMMA 3. If the composition operator $C_{\varphi}$ is isometric on the Bloch space then $\varphi(0)=0$ and $\|\varphi\|_{\mathcal{B}}=1$.

ProOF: Let $\varphi(0)=a$ and

$$
\psi(z)=\frac{a-z}{1-\bar{a} z}
$$

By the Schwarz-Pick lemma, we have

$$
\|\psi\|_{B}=|a|+\|\psi\|_{\beta}=|a|+1
$$

and

$$
\left\|C_{\varphi}(\psi)\right\|_{\mathcal{B}}=|\psi(\varphi(0))|+\sup _{z \in D}\left(1-|\varphi(z)|^{2}\right)\left|\psi^{\prime}(\varphi(z))\right| \frac{1-|z|^{2}}{1-|\varphi(z)|^{2}}\left|\varphi^{\prime}(z)\right| \leqslant 1 .
$$

If $C_{\varphi}$ is isometric, then $a=0$ and $\|\varphi\|_{B}=1$. The lemma is proved.

Theorem 3. Suppose that the composition operator $C_{\varphi}$ is isometric on the Bloch space. Then 
(1) $\varphi^{\prime}$ is bounded on $\mathbf{D} \Longrightarrow \varphi(z)=e^{i \theta} z$.

(2) $\varphi$ is univalent on $\mathbf{D} \Longrightarrow \varphi(z)=e^{i \theta} z$.

(3) If $\varphi(z) \neq e^{i \theta} z$, then for every $a \in \mathbf{D}$ there exists a sequence $\left\{z_{n}\right\}$ such that $\left|z_{n}\right| \rightarrow 1$ and $\varphi\left(z_{n}\right) \rightarrow a$.

Proof: (1) For the isometry of $C_{\varphi}$, we know $\|\varphi\|_{\mathcal{B}}=1$ and $\varphi(0)=0$ by Lemma 3 , hence

$$
\sup _{z \in \mathbf{D}}\left(1-|\varphi(z)|^{2}\right) \cdot \frac{1-|z|^{2}}{1-|\varphi(z)|^{2}}\left|\varphi^{\prime}(z)\right|=1 .
$$

If $\varphi(z) \neq e^{i \theta} z$, by the Schwarz-pick lemma, we have $\tau_{\varphi}(z)<1$ for $z \in \mathbf{D}$. Then there exists a sequence $\left\{z_{n}\right\}$ such that $\left|z_{n}\right| \rightarrow 1,\left|\varphi\left(z_{n}\right)\right| \rightarrow 0$ and $\tau_{\varphi}\left(z_{n}\right) \rightarrow 1$. Hence

$$
\left|\varphi^{\prime}\left(z_{n}\right)\right|>\frac{1-\left|\varphi\left(z_{n}\right)\right|^{2}}{2\left(1-\left|z_{n}\right|^{2}\right)} \rightarrow \infty
$$

for the sufficient large $n$. This contradicts the fact that that $\varphi^{\prime}$ is bounded on $\mathbf{D}$.

(2) Assuming that $\varphi$ is univalent, we know $\varphi(0)=0$ by lemma 3. Then there exists a $\varepsilon>0$ such that $\left|\varphi\left(z_{n}\right)\right|>\varepsilon$ for any sequence $\left|z_{n}\right| \rightarrow 1$ with the sufficient large $n$. If $\varphi(z) \neq e^{i \theta} z$, by the proof of $(1)$, we have a contradiction.

(3) For any $a \in \mathrm{D}$, we take $\psi(z)=(a-z) /(1-\bar{a} z)$. By the Schwarz-Pick lemma and the lemma 3 , we have

$$
\begin{gathered}
|a|+\sup _{z \in \mathbf{D}}\left(1-|\varphi(z)|^{2}\right)\left|\psi^{\prime}(\varphi(z))\right| \cdot \frac{1-|z|^{2}}{1-|\varphi(z)|^{2}}\left|\varphi^{\prime}(z)\right|=|a|+1, \\
\sup _{z \in \mathbf{D}}\left(1-|\psi(\varphi(z))|^{2}\right) \cdot \frac{1-|z|^{2}}{1-|\varphi(z)|^{2}}\left|\varphi^{\prime}(z)\right|=1 .
\end{gathered}
$$

Then there exists a sequence $\left\{z_{n}\right\}$ such that $\left|z_{n}\right| \rightarrow 1$ and $\left|\psi\left(\varphi\left(z_{n}\right)\right)\right| \rightarrow 0$, so $\varphi\left(z_{n}\right) \rightarrow a$.

The proof is completed.

This theorem suggests the following question.

QUESTION. Does there exist a function $\varphi$ other than $e^{i \theta} z$ such that $C_{\varphi}$ is isometric on the Bloch space?

\section{REFERENCES}

[1] J.M. Anderson, J. Clunie and Ch. Pomerenke, 'On Bloch functions and normal functions', J. Reine Angew.Math. 270 (1974), 12-37.

[2] J.E. Littlewood, 'On inequalities in the theory of functions', Proc. London Math. Soc. 23 (1925), 481-519.

[3] E.A. Nordgren, 'Composition operators', Canad. J. Math. 20 (1968), 442-449.

[4] K. Madigan and A. Matheson, 'Compact composition operators on Bloch space', Trans. Amer. Math. Soc. 347 (1995), 2679-2687. 
[5] J.H. Shapiro, 'What do composition operators know about inner functions?', Monatshefte für Mathematik 130 (2000), 57-70.

[6] J.H. Shapiro, Composition operators and classical function theory (Springer-Verlag, New York, 1993), pp. 55-60.

Department of Mathematics

Shantou University

Shantou

Guangdong 515063

Peoples Republic of China
Department of Mathematics

Huaiyin Teacher's College

Huaiyin

Jiangsu 223001

Peoples Republic of China

e-mail: chengji_xiong@yahoo.com 